\title{
Multiexciton generation assisted highly photosensitive CdHgTe nanocrystal skins
}

\author{
Shahab Akhavan a,1, Ahmet Fatih Cihan ${ }^{\mathrm{a}, \mathrm{b}, 1}$, Aydan Yeltik ${ }^{\mathrm{a}}$, Berkay Bozok ${ }^{\mathrm{a}}$, \\ Vladimir Lesnyak $^{\mathrm{c}, \mathrm{d}}$, Nikolai Gaponik ${ }^{\mathrm{d}}$, Alexander Eychmüller ${ }^{\mathrm{d}}$, Hilmi Volkan Demir ${ }^{\mathrm{a}, \mathrm{e}, *}$ \\ ${ }^{a}$ UNAM-Institute of Materials Science and Nanotechnology, Department of Electrical and Electronics Engineering and Department of Physics, Bilkent \\ University, Ankara 06800, Turkey \\ ${ }^{\mathrm{b}}$ Department of Electrical and Electronics Engineering, Stanford University, Stanford, CA 94305, United States \\ ${ }^{\mathrm{c}}$ Department of Nanochemistry, Istituto Italiano di Tecnologia, Genova 16163, Italy \\ d Physical Chemistry, Technische Universität Dresden, Dresden 01062, Germany \\ e LUMINOUS! Center of Excellence, School of Electrical and Electronic Engineering and School of Physical and Mathematical Sciences, Nanyang Technological \\ University, Singapore 639798, Singapore
}

\section{A R T I C L E I N F O}

Article history:

Received 26 December 2015

Received in revised form

8 March 2016

Accepted 28 April 2016

Available online 10 May 2016

Keywords:

Quantum dots

Nanocrystalline materials

Multiexciton generation

Light sensing

Time-resolved fluorescence

\begin{abstract}
A B S T R A C T
Multiexciton Generation (MEG) enabled by the photogeneration of more than one electron-hole pairs upon the absorption of a single photon observed in colloidal semiconductor nanocrystals (NCs) is an essential key to high efficiency when operating in large enough photon energy regimes. Here, we report a newly designed class of solution-processed highly sensitive MEG-assisted photosensors of CdHgTe NCs, in which the charge accumulation is dramatically enhanced for photon energies greater than two times the bandgap of the employed NCs. We fabricated and comparatively studied five types of devices based on different NC monolayers of selected quantum-confined bandgaps resulting in different levels of photovoltage buildup readouts. Among these photosensitive platforms, MEG is distinctly observed for CdHgTe NCs, as the number of electrons trapped inside these NCs and the number of holes accumulating into the interfacing metal electrode were increased beyond a single exciton per absorbed photon. Furthermore, we conducted time-resolved fluorescence measurements and confirmed the occurrence of MEG in the CdHgTe NC monolayer of the photosensor. These findings pave the way for engineering of multiexciton kinetics in high-efficiency NC-based photosensors and photovoltaics.
\end{abstract}

(c) 2016 Elsevier Ltd. All rights reserved.

\section{Introduction}

The creation of a single electron-hole pair per incident photon (single exciton generation) is a fundamental limit for optical absorption and photogeneration in the linear regime. In principle, high-energy photons can create hot excitons with excess energy above the band edge. However, this energy is commonly dissipated as heat through the electron-phonon coupling and subsequent phonon relaxation mechanisms. On the other hand, multiexciton generation (MEG) can exceed the limitation of single exciton generation upon the absorption of a single photon if this excess energy of the photogenerated hot exciton is used to excite a second electron across the bandgap [1-5]. Owing to the favorable

\footnotetext{
*Corresponding author at: UNAM-Institute of Materials Science and Nanotechnology, Department of Electrical and Electronics Engineering and Department of Physics, Bilkent University, Ankara 06800, Turkey.

E-mail addresses: volkan@stanfordalumni.org, hvdemir@ntu.edu.sg (H.V. Demir).

${ }^{1}$ S.A. and A.F.C. contributed equally to this work.
}

excess energy utilization aspect of MEG, its usage in semiconductor nanocrystals (NCs) has particularly attracted significant interest to enhance the performance of solution-processed NCbased optoelectronic devices including photodetectors [6-8] and photovoltaic devices [9-12].

Energy conservation principle dictates that the incoming photon energy needs to be at least twice the bandgap energy of the absorbing material to generate biexcitons (BXs) from a single photon absorption event. Considering this phenomenon and the limited photonic energy of the sunlight spectrum, it is apparent that chemically synthesized NCs with a proper absorption spectrum may open new opportunities for optoelectronic applications [13-18]. Therefore, as an important type of optoelectronic devices, NC-based photosensors utilizing the concept of MEG should include NCs with a bandgap in the near-infrared (NIR), which allows lower threshold energy for more efficient utilization of the sunlight [19]. To date, MEG has been investigated in variety of NCs [20-28] and extensively in lead chalcogenide NCs because of their large exciton Bohr radius in bulk and high degeneracy of the first excited state [29-33]. On the other hand, $\mathrm{Cd}_{\mathrm{x}} \mathrm{Hg}_{1-\mathrm{x}} \mathrm{Te} \mathrm{NCs}$ can also 
be considered as a promising candidate for such studies since the composition adjustability brings another degree of freedom on the design of the electronic structure of NCs [28]. In addition, CdHgTe NCs do not have a long-term stability problem that Pb-based NCs commonly do. Furthermore, CdHgTe NCs can offer unique capabilities including bandgap tunability from visible to NIR region [34], relatively high photoluminescence quantum yields [35], and ease of their colloidal synthesis at low cost and ease of integration on various substrates using dip coating method to form closepacked monolayer semiconducting films [19,36-38], To this end, in this work we investigated the MEG concept in CdHgTe NC platforms, in which the photovoltage buildup of these NCs has been employed. To monitor MEG, photosensitive nanocrystal skin (PNS) devices made of colloidal NC monolayers were fabricated and systematically studied. These light-sensitive NC platforms are promising for photosensing because of their simple and efficient operation on the basis of photogenerated potential buildup across a single NC monolayer without requiring any external bias subject to the close interaction between these NCs and the interfacing metal contact. Device operation is explained in detail in our earlier works using visible-range CdTe NCs operating with single exciton generation [39,40].

Here, we show significant voltage buildup enhancement via MEG because of the increase in electron-hole pair generation and resultant charge accumulation in the CdHgTe NC layer of the PNS. Through the MEG phenomenon in the fabricated PNS devices, a larger number of electrons are trapped inside the NCs while a larger number of holes accumulating at the metal contact. For a comparative study, we fabricated five types of devices with photovoltage readout, using different types of NCs. First, two differentsized CdHgTe NCs with favorable bandgaps possibly allowing for MEG were synthesized and integrated into the PNS platforms for the investigation of sensitivity enhancement via MEG in active device environment. Second, we used the PNS devices hybridized with three different-sized CdTe NCs as the control samples, in which MEG cannot possibly occur in the operating range due to the improper bandgap of these NCs.

\section{Experimental section}

\subsection{Device fabrication and characterization}

ITO film deposited on a glass substrate was washed in a solution of $2 \mathrm{~mL}$ Hellmanex in $100 \mathrm{~mL}$ Milli-Q water for $20 \mathrm{~min}$ using ultrasonication, followed by $20 \mathrm{~min}$ in water, $20 \mathrm{~min}$ in acetone and $20 \mathrm{~min}$ in isopropanol. Following, $50 \mathrm{~nm}$ thick $\mathrm{HfO}_{2}$ dielectric film was deposited using ALD. Subsequently, in order to deposit NCs, we used layer-by-layer self-assembly with a computerized system. Lastly, a $100 \mathrm{~nm} \mathrm{Al} \mathrm{contact} \mathrm{layer} \mathrm{was} \mathrm{laid} \mathrm{down} \mathrm{on} \mathrm{top} \mathrm{of}$ NCs monolayers via a thermal evaporator. We performed all electrical and optical characterizations using Agilent Technologies B1500A semiconductor parameter analyzer without applying any external bias. A Xenon lamp with a monochromator was used as a light source. In order to determine the illumination intensity, a Newport 1835C multi-function optical power meter was employed. During the measurements, samples were grounded from ITO side and connected with a load resistance of $200 \mathrm{M} \Omega$ to the both sides of the ITO and Al contacts.

\subsection{Layer-by-layer assembly}

The layer-by-layer assembly relies on alternating adsorption of positively (PDDA) and negatively (PSS) charged polyelectrolyte pairs. The concentrations of these polyelectrolytes were $2 \mathrm{mg} / \mathrm{mL}$ in $0.1 \mathrm{M} \mathrm{NaCl}$ solution. The cycling procedure was adjusted as

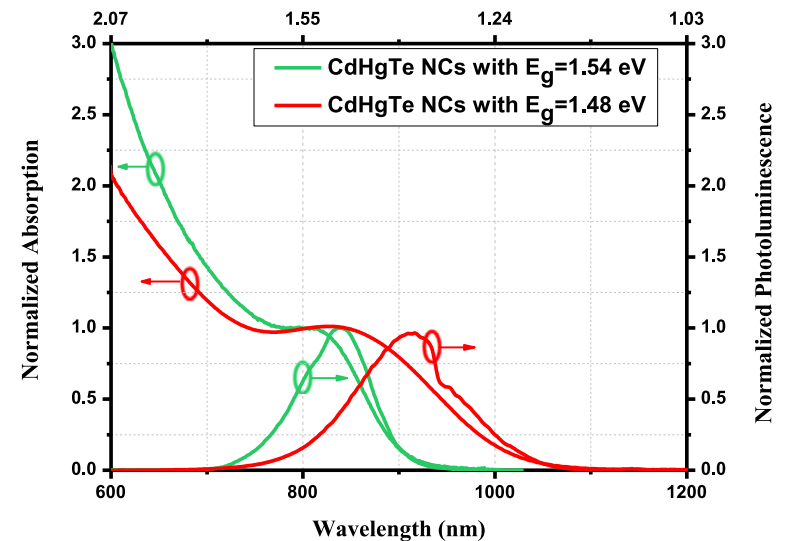

Fig. 1. Absorption and photoluminescence spectra of aqueous CdHgTe NCs at room temperature.

follows: dipping of $\mathrm{HfO}_{2}$-coated substrate into a PDDA solution for $5 \mathrm{~min}$, followed by washing it with water for $1 \mathrm{~min}$, next dipping it into a solution of PSS for $5 \mathrm{~min}$, and washing it with water for $1 \mathrm{~min}$. This procedure was continued until four bilayers of PDDA and PSS composite were obtained. Finally, after deposition of one extra layer of PPDA, sample dipped into the CdTe NCs solution for $20 \mathrm{~min}$ and rinsed with water again for $1 \mathrm{~min}$.

\subsection{Time-resolved fluorescence measurements}

The decay curves were fitted using one and two exponential decays $\left(\chi^{2}-1\right)$ that led to the best $\chi^{2}$ values and the excited-state lifetimes for the samples were calculated via amplitude-averaging. TRF experiments were conducted using Horiba Jobin Yvon TCSPC setup (FL 1057) with pulsed excitation, variable-wavelength LED sources. In the TRF measurements, concentration of the CdHgTe NC samples was kept at minimum within the experimentally feasible region in order to avoid nonradiative energy transfer among the NCs in the ensemble. The dispersion samples were vigorously stirred during measurements to avoid photocharging of the NCs which may cause misleading Auger Recombination signatures in the decay curves.

\subsection{Synthesis of nanocrystals}

All the chemicals used were of analytical grade or of the highest purity available. All the solutions were prepared using Milli-Q water (Millipore) as a solvent. In order to synthesize CdTe NCs, $4.59 \mathrm{~g}$ of $\mathrm{Cd}\left(\mathrm{ClO}_{4}\right)_{2} \times 6 \mathrm{H}_{2} \mathrm{O}$ was dissolved in $500 \mathrm{~mL}$ of Milli-Q water followed by addition of $1.33 \mathrm{~g}$ of thioglycolic acid (TGA) and adjustment of the $\mathrm{pH}$ to $11.8-12.0$. After that, we introduced $\mathrm{H}_{2} \mathrm{Te}$ gas by reacting $0.8 \mathrm{~g} \mathrm{Al}_{2} \mathrm{Te}_{3}$ with $\mathrm{H}_{2} \mathrm{SO}_{4}$ with a slow Ar flow using a setup shown in Lesnyak et al. [38]. At $100{ }^{\circ} \mathrm{C}$, the nucleation and growth of the NCs was initiated. The reaction mixture was boiled under reflux until reaching a desired NC size. Afterwards, the NCs were precipitated by concentration of the reaction mixture on a rotor evaporator (evaporating approx. 90\% of water) followed by addition of $2-3 \mathrm{~mL}$ of isopropanol (as a non-solvent) with subsequent centrifugation of the resulting turbid mixture, and then the precipitated NCs were redissolved in $5-10 \mathrm{~mL}$ of Milli- $\mathrm{Q}$ water. $\mathrm{Cd}_{\mathrm{x}} \mathrm{Hg}_{1-\mathrm{x}} \mathrm{Te} \mathrm{NCs}$ were synthesized employing $0.545 \mathrm{~g}$ of $\mathrm{Cd}\left(\mathrm{ClO}_{4}\right)_{2} \times 6 \mathrm{H}_{2} \mathrm{O}, 0.0135 \mathrm{~g}$ of $\mathrm{Hg}\left(\mathrm{ClO}_{4}\right)_{2} \times 6 \mathrm{H}_{2} \mathrm{O}$ and $0.183 \mathrm{~g}$ of mercaptopropionic acid (MPA) dissolved in $60 \mathrm{~mL}$ of water. Then, in order to adjust the $\mathrm{pH}$ up to $12,1 \mathrm{M} \mathrm{NaOH}$ solution was added. After all, $0.15 \mathrm{~g}$ of $\mathrm{Al}_{2} \mathrm{Te}_{3}$ was used to generate $\mathrm{H}_{2} \mathrm{Te}$ gas. Subsequently, the molar ratio of $\mathrm{Cd}^{2+} / \mathrm{Hg}^{2+} / \mathrm{Te}^{2-} / \mathrm{MPA}$ was $0.98 / 0.02 /$ $0.75 / 1.3$. The color of the solution turned to brown after injection of $\mathrm{H}_{2}$ Te. Similar to the case of CdTe NCs, nucleation and growth of 

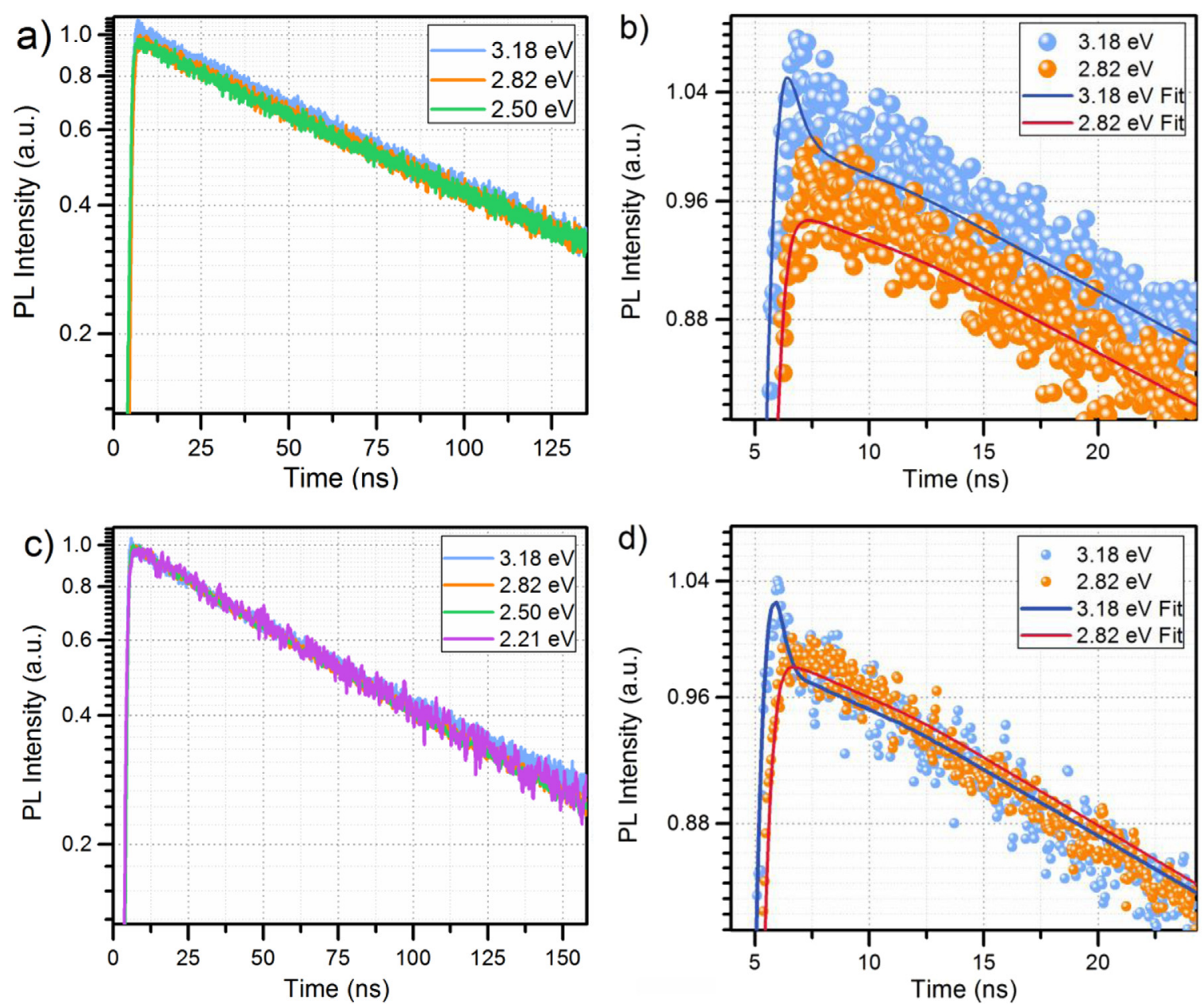

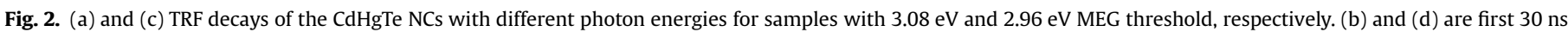

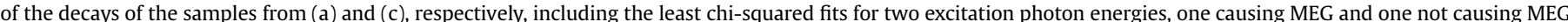
for samples with $3.08 \mathrm{eV}$ and $2.96 \mathrm{eV}$ MEG threshold, respectively.

Table 1

TRF amplitudes and lifetimes from the optical measurements of CdHgTe NCs with multiexciton generation threshold at 3.08 and $2.96 \mathrm{eV}$.

\begin{tabular}{|c|c|c|c|c|c|c|c|c|}
\hline \multirow[t]{2}{*}{ Photon energy (eV) } & \multicolumn{4}{|l|}{$3.08 \mathrm{eV}$} & \multicolumn{4}{|l|}{$2.96 \mathrm{eV}$} \\
\hline & $A_{1}$ & $\tau_{1}(\mathrm{~ns})$ & $A_{2}$ & $\tau_{2}(\mathrm{~ns})$ & $A_{1}$ & $\tau_{1}(\mathrm{~ns})$ & $A_{2}$ & $\tau_{2}(\mathrm{~ns})$ \\
\hline \multirow[t]{2}{*}{3.18} & $1.1 \times 10^{-2}$ & 104.4 & $5.3 \times 10^{-2}$ & 0.1 & $9.2 \times 10^{-1}$ & 106.3 & $6.5 \times 10^{-1}$ & 0.5 \\
\hline & $\left( \pm 7.3 \times 10^{-6}\right)$ & $\left( \pm 194.2 \times 10^{-3}\right)$ & $\left( \pm 8.9 \times 10^{-4}\right)$ & $\left( \pm 11.7 \times 10^{-3}\right)$ & $\left( \pm 5.5 \times 10^{-3}\right)$ & $\left( \pm 101.2 \times 10^{-2}\right)$ & & \\
\hline \multirow[t]{2}{*}{2.82} & $1.6 \times 10^{-2}$ & 107.0 & $8.6 \times 10^{-3}$ & 0.1 & $9.4 \times 10^{-1}$ & 106.4 & - & - \\
\hline & $\left( \pm 6.8 \times 10^{-4}\right)$ & $\left( \pm 125.3 \times 10^{-3}\right)$ & $\left( \pm 6.6 \times 10^{-4}\right)$ & $\left( \pm 7.4 \times 10^{-3}\right)$ & $\left( \pm 5.3 \times 10^{-3}\right)$ & $\left( \pm 102.3 \times 10^{-2}\right)$ & & \\
\hline \multirow[t]{2}{*}{2.50} & $5.8 \times 10^{-5}$ & 104.6 & - & - & $7.0 \times 10^{-1}$ & 100.1 & - & - \\
\hline & $\left( \pm 4.0 \times 10^{-6}\right)$ & $\left( \pm 188.9 \times 10^{-3}\right)$ & & & $\left( \pm 5.7 \times 10^{-3}\right)$ & $\left( \pm 133.9 \times 10^{-2}\right)$ & & \\
\hline \multirow[t]{2}{*}{2.21} & $1.6 \times 10^{-3}$ & 104.0 & - & - & - & - & - & - \\
\hline & $\left( \pm 4.7 \times 10^{-6}\right)$ & $\left( \pm 714.2 \times 10^{-3}\right)$ & & & & & & \\
\hline
\end{tabular}

$\mathrm{Cd}_{\mathrm{x}} \mathrm{Hg}_{1-\mathrm{x}} \mathrm{Te} \mathrm{NCs}$ proceeded upon ambient conditions. After cooling the $\mathrm{NC}$ solutions to room temperature, they were purified in the same way as CdTe NC colloids.

\section{Results and discussion}

The goal is to use the excess energy of a high-energy photon to generate an extra electron-hole pair via MEG in a NC, thus increasing the resulting efficiency at the device level at the next stage $[6,41]$. To reveal this phenomenon, time-resolved fluorescence (TRF) spectroscopy is preferred and extensively used as a powerful technique for a detailed analysis of multiexciton recombination dynamics in NCs. Excitation photon energy threshold for the MEG process is calculated using the relation $\hbar \omega_{t h}=\left(2+m_{e} / m_{h}\right) E_{g}$, where $m_{e}$ and $m_{h}$ are the effective masses of electron and hole, respectively, and $E_{g}$ is the bandgap energy of the light absorbing material $[1,42,43]$. According to this expression, MEG threshold should be higher than twice the bandgap energy of 


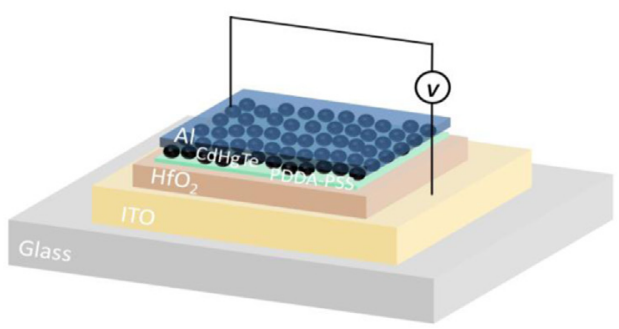

$\mathbf{E}$

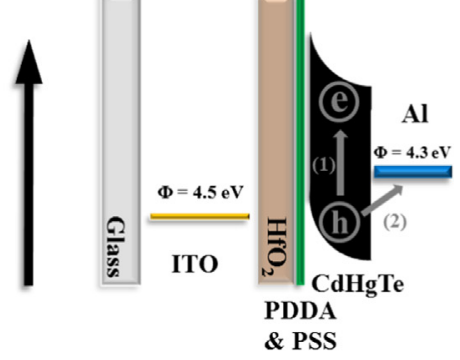

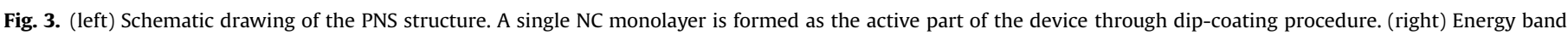

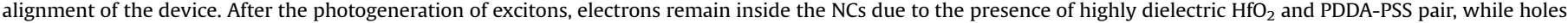
migrate to the Al electrode layer due to the work function of the NCs and Al contact.
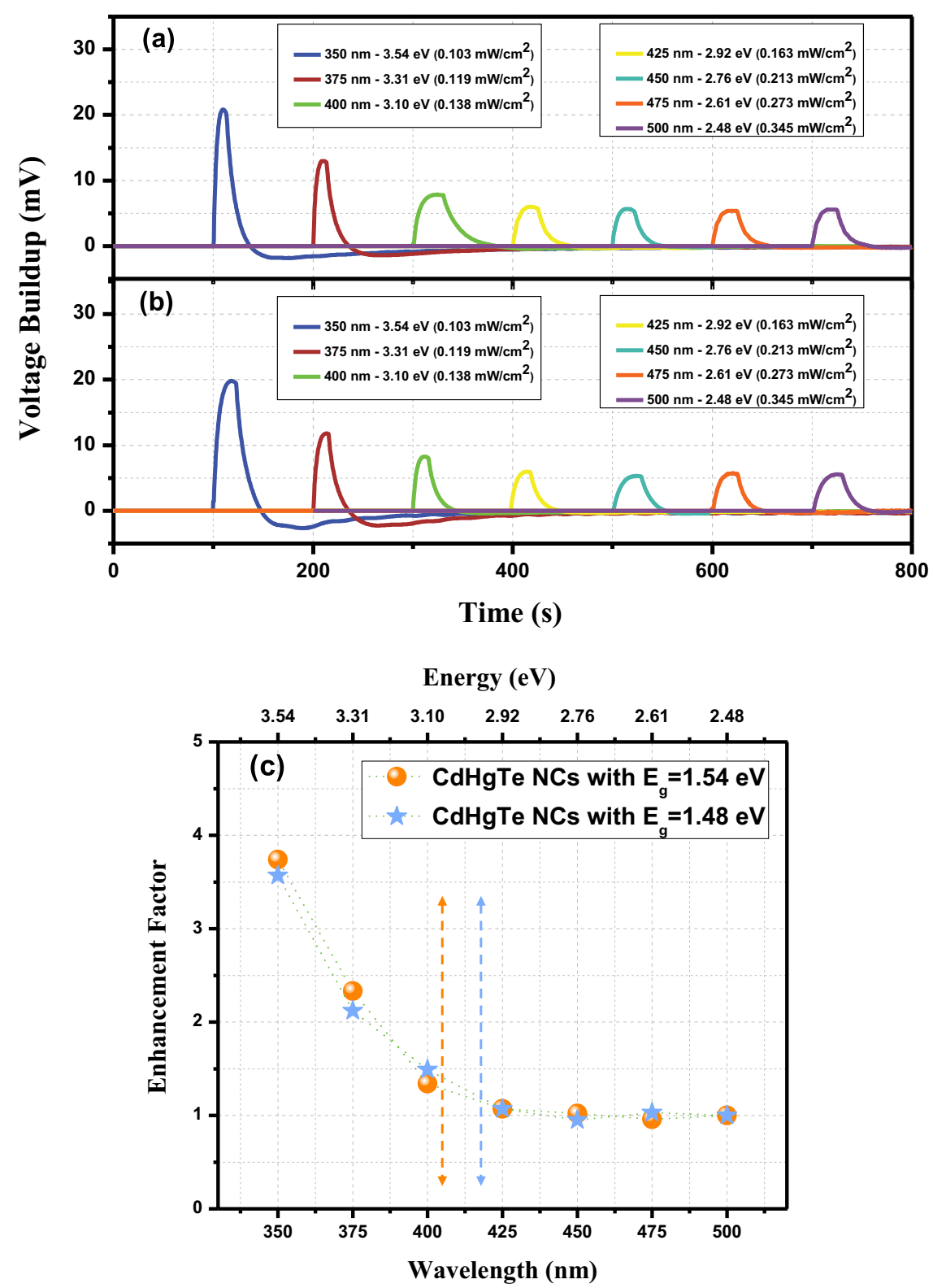

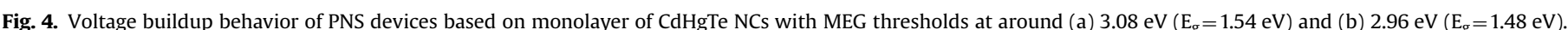

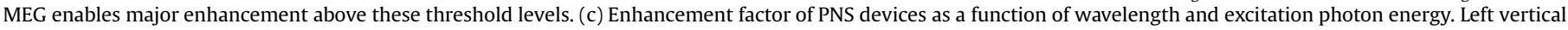

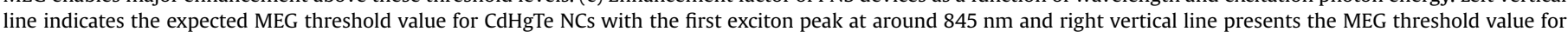
CdHgTe NCs with the first exciton peak at around $805 \mathrm{~nm}$.

the NC since MEG occurs only when one of the charge carriers has kinetic energy as large as the bandgap energy. CdHgTe NCs are advantageous in terms of the MEG threshold because the hole effective mass is much bigger than the electron effective mass, leading to $\hbar \omega_{t h} \approx 2 E_{g}$. For this reason, we synthesized two different sets of CdHgTe NCs with their first excitonic peaks around $805 \mathrm{~nm}$ 


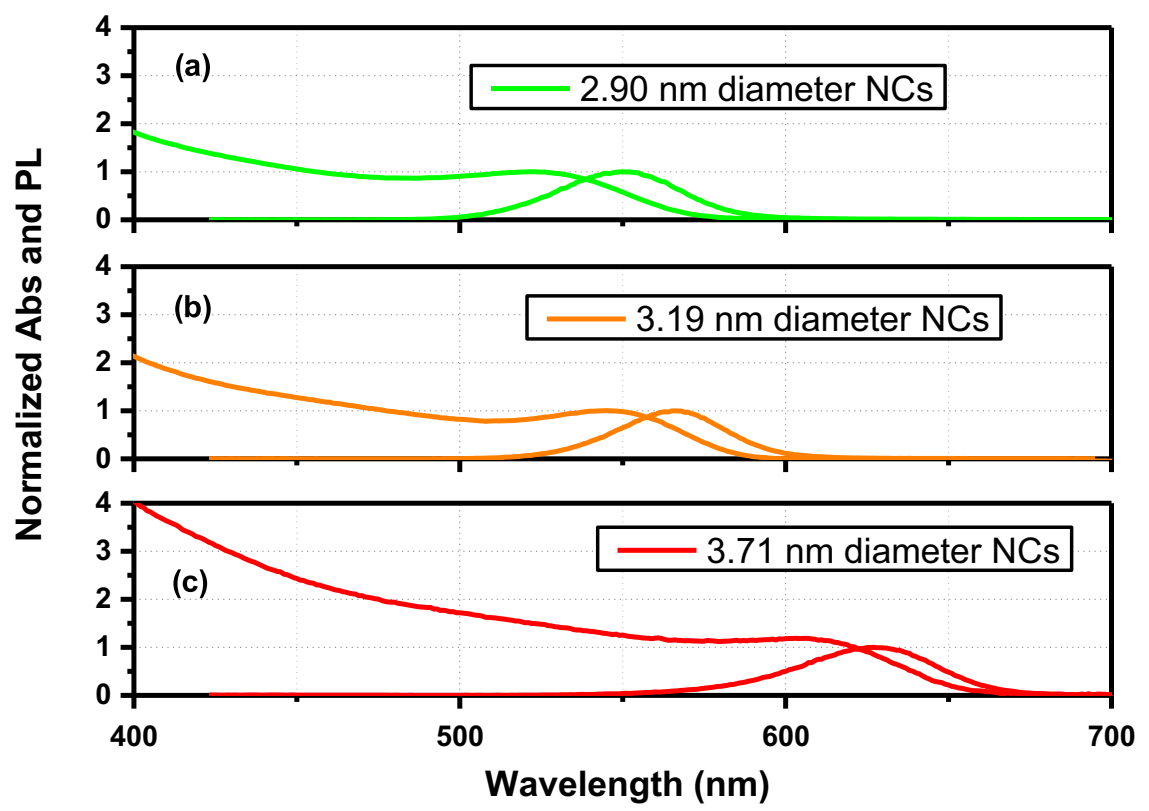

Fig. 5. Absorption and photoluminescence spectra of the as-synthesized aqueous CdTe NC solutions with diameters of (a) $2.90 \mathrm{~nm}$, (b) $3.19 \mathrm{~nm}$, and (c) $3.71 \mathrm{~nm}$.

(bandgap of $1.54 \mathrm{eV}$ ) and $840 \mathrm{~nm}$ (bandgap of $1.48 \mathrm{eV}$ ). Subsequently, the MEG thresholds expected in our experiments with these NCs are about 3.08 and $2.96 \mathrm{eV}$, respectively. Fig. 1 demonstrates the photoluminescence and optical absorption spectra of these NCs. Transition electron microscopy images of these NCs are also presented in Fig. S1.

BXs generated in CdHgTe NCs as a result of the MEG process are expected to decay nonradiatively via Auger recombination (AR), which has a lifetime that is two to three orders of magnitude shorter than that of the radiative recombination in these NCs. Therefore, the presence of this very fast AR decay term in the global decay of NCs is to be considered as a signature of any MEG event, which is the case for many reports in the literature [44-47]. It is very critical to note that BXs are not the only ones that recombine via AR, as trions can also recombine through AR. This can cause a false MEG signature in the TRF decays obtained from the optical measurements. In this study, we vigorously stirred the NC colloid during the measurements to avoid photocharging, and hence the generation of trions. We observed that the same TRF decays appeared which means that the photocharging effect was not a significant issue for our results.

To verify the existence of MEG, we conducted TRF measurements under various excitation photon energies as provided in Fig. 2. In order to avoid generation of BXs by multiple photon absorption events and ensure that the generated BXs are only from single photon absorption events, we kept the excitation intensity as low as possible during the experiments. The emergence of the very short lifetime component due to the multiexciton recombination process for the cases with sufficient photon energies is not so obvious in Fig. 2(a) and (c). A closer look at the first $30 \mathrm{~ns}$ part of the decays in Fig. 2(b) and (d) clearly reveals the existence of fast decay term for the excitation photon energy of $3.18 \mathrm{eV}$. The fast decay term is not observed for the case with the excitation photon energy of $2.82 \mathrm{eV}$ as expected because the MEG threshold value, $2.96 \mathrm{eV}$, is greater than the photon energy. This consistency between MEG threshold expectation and the experimental photon energy threshold for the fast decay peak suggests that this fast decay component results from the MEG of CdHgTe NCs.

Recombination lifetimes extracted from the TRF curves are presented in Table 1. An important point that should be noted is that the pulse widths of the excitation sources are 1.3-1.4 ns while the fast decay lifetime that we attribute to AR is expected to be on the order of hundreds of picoseconds. As a result, the wide pulse of the excitation LED smears out the fast decay component. To extract the lifetime of the fast decay component and make reliable conclusions, TRF decays were fitted using the reconvolution mode of the fitting program (FluoFit-PicoQuant). It was observed that for all the excitation photon energy cases the lifetime values of the slow and fast decay processes are similar, which are $\sim 104 \mathrm{~ns}$, and $\sim 116$ ps, respectively. Here, the long-lifetime decay term belongs to single exciton recombination in the NCs and the short lifetime can be attributed to the AR of the BXs created in the NCs. The AR lifetime of the BXs turned out to be sub-nanosecond as expected. Also, the amplitude weight of the fast decay component, $A_{2}$, with respect to the coefficient of slow decay component, $A_{1}$, is larger for the photon energy of $3.18 \mathrm{eV}$ while it is smaller for the photon energy of $2.82 \mathrm{eV}$. This means that the increase in photon energy also increases the MEG probability as would be intuitively expected.

Afterwards, the PNS devices were fabricated for proof-of-concept demonstration of the MEG concept in an active photosensor platform. For this purpose, $50 \mathrm{~nm}$ thick highly dielectric $\mathrm{HfO}_{2}$ was deposited on top of indium tin oxide (ITO) substrate via atomic layer deposition (ALD) over $1 \mathrm{~cm} \times 0.75 \mathrm{~cm}$ active area. With the help of water pulses used in ALD, a hydrophilic surface was preferentially formed, which helps to improve the quality of dipcoated NC film on top of it. Subsequently, in order to deposit highly close-packed monolayer of CdHgTe NCs on the substrate, poly (diallyldimethylammonium chloride) (PDDA) and poly (sodium 4-styrenesulfonate) (PSS) bilayers were deposited in an alternating fashion to build four bilayers using layer-by-layer assembly [48]. Finally, Al contact was laid down on top of the NC monolayer by using thermal evaporator in a vacuum environment. After the device fabrication, voltage-time (V-t) measurements were performed to characterize the device without applying any external bias. During these measurements, samples were grounded from the ITO contact and connected in series with a load resistance of $200 \mathrm{M} \Omega$.

Device architecture and band diagram of the proposed device structure are shown in Fig. 3. The light incident on the device leads to electron-hole pairs generation. Because of the work functions of the NCs monolayer and Al layer [49], holes accumulate at the Al 

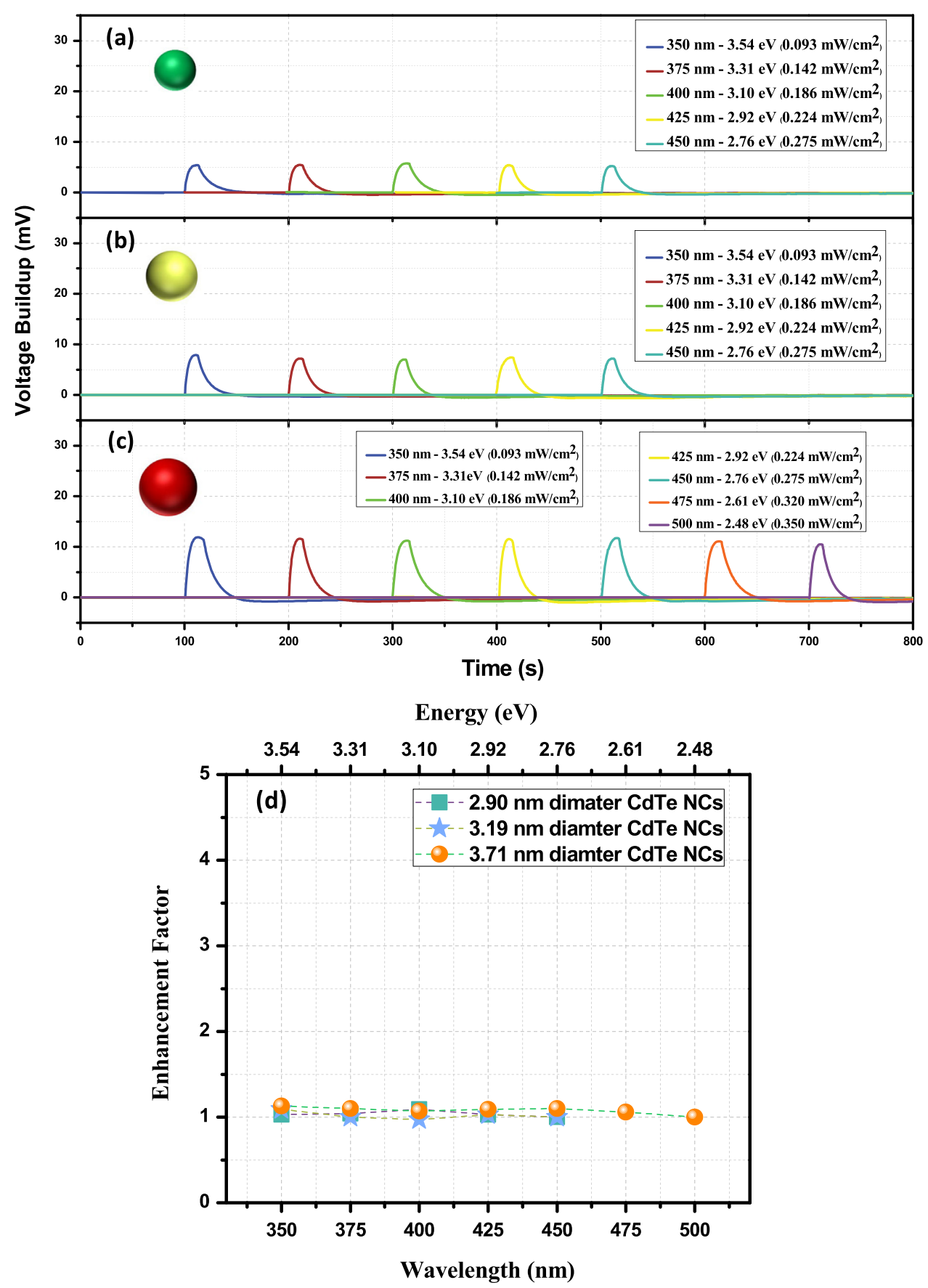

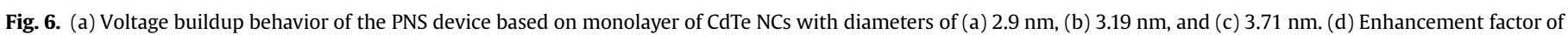
PNS devices as a function of wavelength and excitation photon energy based on different sized CdTe NCs.

contact, generating a positive potential buildup whereas most of the electrons remain inside the NCs due to the blockage by $\mathrm{HfO}_{2}$ layer. The amount of positive charge accumulation at the metal contact and negative internal voltage buildup are directly related to the number of photogenerated holes and electrons [50], After reaching the peak point in the voltage buildup, the incident light is switched off and the voltage buildup is diminished. After a certain short period of time, the potential buildup goes negative due to the trapped electrons inside the NCs, which also decays in time, and the device finally recovers back to its initial state.

To measure the effect of MEG on photosensing performance, PNSs were illuminated with Xenon lamp light source with a monochromator as a tunable-wavelength source. During the voltage buildup measurements, the number of absorbed photons impinging on the device at each wavelength was adjusted keeping in mind the absorbance of NCs and photon energy at the corresponding wavelength. Consequently, in each device, the number of absorbed photons was set to be identical throughout the measured wavelengths. As it can be seen, the voltage buildup graphs of CdHgTe NCs shown in Fig. 4(a) and (b) reveal no change, approximately remaining constant, up to the MEG threshold value. However, for energies larger than the MEG threshold value, $2.96 \mathrm{eV}$ and $3.08 \mathrm{eV}$, the voltage buildup rises sharply. Evidently, at the excitation wavelength of $350 \mathrm{~nm}$, the output voltage corresponding to longer wavelengths where MEG does not occur is enhanced by $\sim 3.57$ folds for the CdHgTe NCs with the first exciton peak at around $845 \mathrm{~nm}$ and similarly $\sim 3.74$ folds enhancement for the one with the first exciton peak at around $805 \mathrm{~nm}$, see Fig. 4(c). 
Observation of this high photovoltage buildup of the device indicates that there is a significant enhancement in the charge generation and subsequent hole accumulation processes for photon energies greater than the MEG threshold.

For comparison purposes, we also fabricated PNS devices using monolayer CdTe NCs having large enough bandgap energy that prevents MEG. To this end, we conducted the voltage buildup measurements with different-sized CdTe NCs, which are extensively used as common quantum confined nanostructures in the visible range in the literature [51-53]. We used CdTe NCs with the diameters of 2.9, 3.19 and $3.71 \mathrm{~nm}$ (calculated from their extinction spectra $[54,55]$ ) leading to the first excitonic peak wavelengths of 525,545 and $605 \mathrm{~nm}$, respectively. The photoluminescence and absorption spectra of the CdTe NCs with various sizes are depicted in Fig. 5 as the control group of this study.

Accordingly, none of these CdTe NCs based devices showed any significant increase in the photovoltage buildup or sensitivity at any photon energy, see Fig. 6. Indeed, voltage buildups remain constant from longer wavelengths to shorter ones because of improper bandgap energy of CdTe NCs for the MEG. Furthermore, it can be easily seen that larger NCs have higher voltage buildup. The first possible reason for that is the quantum confinement. Larger NCs have larger number of states available, which result in higher optical absorption and thus higher voltage buildup. Secondly, small-sized NCs generally have a larger number of trap states than large-sized NCs, which hinder the migration of photogenerated positive charges $[56,57]$. Subsequently, the devices with the larger CdTe NCs show better performance and operate at longer wavelengths as it can be clearly seen from Fig. 6 yet with no enhancement (i.e., unity enhancement factor) given in Fig. 6 (d) unlike the MEG assisted PNS devices of CdHgTe shown in Fig. 4.

\section{Conclusion}

In this study, for the first time, we showed MEG-assisted enhancement in a photosensing device using aqueous CdHgTe NCs with the bandgap in the near-infrared region. Significant MEG evidence was revealed in the PNS devices with two different-sized CdHgTe NCs utilizing the enhancement of electrons trapped inside the NCs and the holes migrating into the metal contact per each absorbed photon above the MEG threshold levels. As the further experimental confirmation, we studied the voltage buildup of three different-sized CdTe NCs in control devices. As it was expected, due to the improper bandgap of CdTe NCs for MEG, voltage buildup profile with no enhancement was observed through the operating wavelength range of the device, which indicates the absence of MEG. These findings indicate significant implications for the future colloidal photosensor designs leading to a new generation of highly light-sensitive devices.

\section{Acknowledgement}

This research was financially supported by the Institute of Materials Science and Nanotechnology at Bilkent University and Nanyang Technological University. HVD gratefully acknowledge the financial support in part by ESF EURYI, EU FP7 NoE Nanophotonics for Energy Efficiency, and TUBITAK under the Project No. EEEAG 110E217, 114E449, 114F326, and 112E183 and in part by NRF grants NRF-CRP6-2010-02 and NRF RF 2009-09. HVD acknowledges additional support from TUBA-GEBIP.

\section{Appendix A. Supplementary material}

Supplementary data associated with this article can be found in the online version at http://dx.doi.org/10.1016/j.nanoen.2016.04.055.

\section{References}

[1] A.J. Nozik, Chem. Phys, Lett. 2 (2008) 3-11.

[2] M.C. Beard, J. Phys. Chem. Lett. 2 (2011) 1282-1288.

[3] A.F. Cihan, P.L. Hernandez Martinez, Y. Kelestemur, E. Mutlugun, H.V. Demir, ACS Nano 7 (2013) 4799-4809.

[4] A.F. Cihan, Y. Kelestemur, B. Guzelturk, O. Yerli, U. Kurum, H.G. Yaglioglu, A. Elmali, H.V. Demir, J. Phys. Chem. Lett. 4 (2013) 4146-4152.

[5] C. Sealy, Nano Energy 1 (2012) 191-194.

[6] V. Sukhovatkin, S. Hinds, L. Brzozowski, E.H. Sargent, Science 324 (2009) 1542-1544.

[7] S.W. Shin, K.-H. Lee, J.-S. Park, S.J. Kang, ACS Appl. Mater. Interfaces 7 (2015) 19666-19671.

[8] X. Liu, X. Ji, M. Liu, N. Liu, Z. Tao, Q. Dai, L. Wei, C. Li, X. Zhang, B. Wang, ACS Appl. Mater. Interfaces 7 (2015) 2452-2458.

[9] R. Yu, Q. Lin, S.F. Leung, Z. Fan, Nano Energy 1 (2012) 57-72.

[10] A.J. Nozik, M.C. Beard, J.M. Luther, M. Law, R.J. Ellingson, J.C. Johnson, Chem. Rev. 110 (2010) 6873-6890.

[11] G. Nair, L.Y. Chang, S.M. Geyer, M.G. Bawendi, Nano Lett. 11 (2011) 2145-2151.

[12] J. Patel, F. Mighri, A. Ajji, T.K. Chaudhuri, Nano Energy 5 (2014) 36-51.

[13] E.-H. Kong, S.-H. Joo, H.-J. Park, S. Song, Y.-J. Chang, H.S. Kim, H.M. Jang, Small 10 (2014) 3678-3684.

[14] T. Erdem, H.V. Demir, Nat. Photonics 5 (2011) 126

[15] S. Coe-sullivan, Nat. Photonics 3 (2009) 315-316.

[16] X. Zhang, C. Zhou, S. Zang, H. Shen, P. Dai, X. Zhang, L.S. Li, ACS Appl. Mater. Interfaces 7 (2015) 14770-14777.

[17] F.P. García de Arquer, T. Lasanta, M. Bernechea, G. Konstantatos, Small 11 (2015) 2636-2641.

[18] S.V. Gaponenko, Introduction to Nanophotonics, Cambridge University Press, Cambridge, U.K., 2010.

[19] A.L. Rogach, A. Eychmüller, S.G. Hickey, S.V. Kershaw, Small 3 (2007) 536-557.

[20] M.C. Beard, K.P. Knutsen, P. Yu, J.M. Luther, Q. Song, W.K. Metzger, R. . Ellingson, A.J. Nozik, Nano Lett. 7 (2007) 2506-2512.

[21] S.K. Stubbs, S.J.O. Hardman, D.M. Graham, B.F. Spencer, W.R. Flavell, P. Glarvey, O. Masala, N.L. Pickett, D.J. Binks, Phys. Rev. B 81 (2010) 081303.

[22] G. Allan, C. Delerue, Phys. Rev. B 77 (2008) 125340.

[23] J.E. Murphy, M.C. Beard, A.J. Nozik, J. Phys. Chem. B 110 (2006) 25455-25461.

[24] J.M. Luther, M.C. Beard, Q. Song, M. Law, R.J. Ellingson, A.J. Nozik, Nano Lett. 7 (2007) 1779-17784.

[25] M.C. Beard, A.G. Midgett, M.C. Hanna, J.M. Luther, B.K. Hughes, A.J. Nozik, Nano Lett. 10 (2010) 3019-3027.

[26] R.D. Schaller, M. Sykora, J.M. Pietryga, V.I. Klimov, Nano Lett. 6 (2006) 424-429.

[27] J.E. Murphy, M.C. Beard, A.G. Norman, S.P. Ahrenkiel, J.C. Johnson, P. Yu, O. I. Mićić, R.J. Ellingson, A.J. Nozik, J. Am. Chem. Soc. 128 (2006) 3241-3247.

[28] A. Al-Otaify, S.V. Kershaw, S. Gupta, A.L. Rogach, G. Allan, C. Delerue, D.J. Binks Phys, Chem. Chem. Phys, 15 (2013) 16864-16873.

[29] J.G. Tischler, T.A. Kennedy, E.R. Glaser, A.L. Efros, E.E. Foos, J.E. Boercker, T. J. Zega, R.M. Stroud, S.C. Erwin, Phys. Rev. B 82 (2010) 245303.

[30] A.C. Bartnik, A.L. Efros, W.-K. Koh, C.B. Murray, F.W. Wise, Phys. Rev. B 82 (2010) 195313

[31] R.D. Schaller, V.I. Klimov, Phys. Rev. Lett. 92 (2004) 186601.

[32] R.D. Schaller, M.A. Petruska, V.I. Klimov, Appl. Phys. Lett. 87 (2005) 253102.

[33] X. Zhu, Acc. Chem. Res. 46 (2013) 1239-12341.

[34] H. Sun, J. Zhang, Y. Tian, Y. Ning, H. Zhang, J. Ju, D. Li, S. Xiang, B. Yang, Chem. Mater. 20 (2008) 6764-6769.

[35] S. Hatami, C. Würth, M. Kaiser, S. Leubner, S. Gabriel, L. Bahrig V. Lesnyak, J. Pauli, N. Gaponik, A. Eychmüller, U. Resch-Genger, Nanoscale 7 (2015) 133-143.

[36] V. Lesnyak, A. Lutich, N. Gaponik, M. Grabolle, A. Plotnikov, U. Resch-genger, A. Eychmüller, J. Mater. Chem. 19 (2009) 9147.

[37] A.L. Rogach, M.T. Harrison, S.V. Kershaw, A. Kornowski, M.G. Burt, A. Eychmüller, H. Weller, Phys. Status Solidi Basic Res. 224 (2001) 153-158.

[38] V. Lesnyak, N. Gaponik, A. Eychmüller, Chem. Soc. Rev. 42 (2013) 2905-2929.

[39] S. Akhavan, B. Guzelturk, V.K. Sharma, H.V. Demir, Opt. Express 20 (2012) 25255-25266.

[40] S. Akhavan, K. Gungor, E. Mutlugun, H.V. Demir, Nanotechnology 24 (2013) 155201.

[41] O.E. Semonin, J.M. Luther, S. Choi, H.-Y. Chen, J. Gao, A.J. Nozik, M.C. Beard (80Science 334 (2011) 1530-1533.

[42] J.J.H. Pijpers, E. Hendry, M.T.W. Milder, R. Fanciulli, J. Savolainen, J.L. Herek, D. Vanmaekelbergh, S. Ruhman, D. Mocatta, D. Oron, A. Aharoni, U. Banin, M. Bonn, J. Phys. Chem. C 111 (2007) 4146-41452.

[43] A. Franceschetti, J.M. An, A. Zunger, Nano Lett. 6 (2006) 2191-2195.

[44] M. Marceddu, M. Saba, F. Quochi, A. Lai, J. Huang, D.V. Talapin, A. Mura, G. Bongiovanni, Nanotechnology 23 (2012) 015201.

[45] F. García-Santamaría, S. Brovelli, R. Viswanatha, J. a Hollingsworth, H. Htoon, S a Crooker, V.I. Klimov, Nano Lett. 11 (2011) 687-693.

[46] F. Garcia-Santamaria, Y. Chen, J. Vela, J.A. Hollingsworth, R.D. Schaller, V. I. Klimov, Nano Lett. 9 (2009) 3482-3488.

[47] V.I. Klimov, J. Phys. Chem. B 110 (2006) 16827-16845.

[48] A. Shavel, N. Gaponik, A. Eychmüller, Eur. J. Inorg. Chem. 2005 (2005) 3613-3623.

[49] S. Akhavan, C. Uran, B. Bozok, K. Gungor, Y. Kelestemur, V. Lesnyak, N. Gaponik, A. Eychmuller, H.V. Demir, Nanoscale 8 (2016) 4495-4503. 
[50] S. Akhavan, A.F. Cihan, B. Bozok, H.V. Demir, Small 10 (2014) 2470-2475.

[51] A.L. Rogach, T. Franzl, T.A. Klar, J. Feldmann, N. Gaponik, V. Lesnyak, A. Shavel, A. Eychmuller, Y.P. Rakovich, J.F. Donegan, J. Phys. Chem. C 111 (2007) 14628-14637.

[52] A. Yeltik, B. Guzelturk, P.L. Hernandez-Martinez, S. Akhavan, H. volkan Demir, Appl. Phys. Lett. 103 (2013) 261103.

[53] Y. Li, L. Jing, R. Qiao, M. Gao, Chem. Commun. 47 (2011) 9293-9311.

[54] P. Reiss, M. Protière, L. Li, Small 5 (2009) 154-168.

[55] W.W. Yu, L. Qu, W. Guo, X. Peng, Chem. Mater. 125 (2003) 2854-2860.

[56] S. Akhavan, A. Yeltik, H.V. Demir, ACS Appl. Mater. Interfaces 6 (2014) 9023-9028.

[57] N. Li, H. Wang, Q. Lin, H. Shen, A. Wang, L. Qian, F. Guo, L.S. Li, RSC Adv. 5 (2015) 39714-39718.

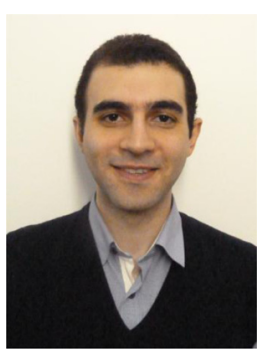

Shahab Akhavan received his B.Eng. degree in Materials Science and Engineering at Middle East Technical University and his M.S. in Materials Science and Nanotechnology at Bilkent University under the supervision of Prof. Dr. Hilmi Volkan Demir. Currently, he is a Ph.D. student in University of Cambridge Engineering Department, Electrical Engineering Division. His main research interests include the design and study on the OD, 1D and 2D nanomaterials for high efficiency photosensors, light-emitting diodes and solar cells.

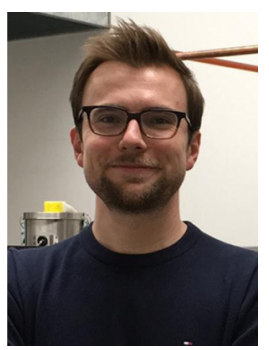

Ahmet Fatih Cihan received his B.S. degree (2010) from Middle East Technical University and M.S. degree (2013) from Bilkent University, both in Electrical Engineering. He is now a Ph.D. student and a research assistant at Stanford University, Electrical Engineering Department. His research interests include quantum dot lasing, multi-exciton generation in nano-crystals, semiconductor/dielectric nano-antennas and light manipulation at the nanoscale.

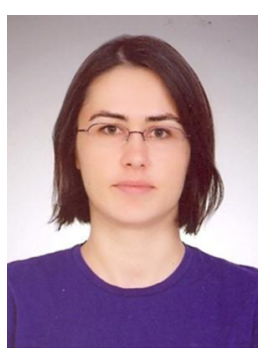

Aydan Yeltik is a Ph.D. candidate at the Department of Physics, Bilkent University, Turkey. She received her B.S. and M.S. degrees in Physics (with honors) from Middle East Technical University, Turkey. Her research activity mainly concerns the study of excitonic interactions in the complex media consisting of $0 \mathrm{D}, 2 \mathrm{D}$ and 3D materials for various applications in optoelectronics.

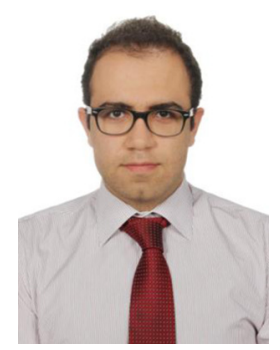

Berkay Bozok was born in Ankara, Turkey on June. 10, 1991. He received his B.S. degree in Electrical and Electronics Engineering and minor degree in Physics from Bilkent University, Ankara, Turkey, in 2014. After that, he started his MS study at the same year in the same Department and University. His main research interest is the design and study on the nano-structured materials for electronic devices.

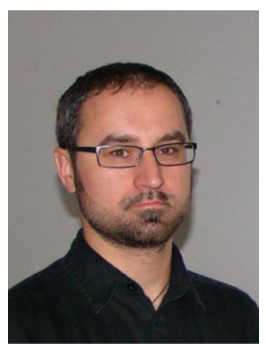

Vladimir Lesnyak received his Ph.D. degree in chemistry (2005) from the Belarusian State University (Minsk, Belarus). Ph.D. studies were followed by postdoctoral research at the Technical University of Dresden (Germany) in the group of Prof. Alexander Eychmüller. Thereafter, he received a Marie Curie fellowship and worked at the Istituto Italiano di Tecnologia (Genoa, Italy) in the group of Prof. Liberato Manna. Currently he is a senior research scientist at the TU Dresden. His research focuses on the synthesis, assembly and applications of colloidal nanomaterials.

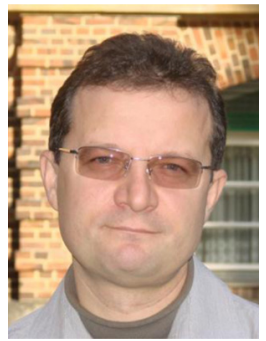

Nikolai Gaponik is a senior staff scientist and ad personam professor at the TU Dresden. He received his $\mathrm{Ph}$. D. in chemistry (2000) from the Belarussian State University in Minsk under supervision of Prof. D. V. Sviridov and made his habilitation (2013) at the TU Dresden. He was a visiting scientist at the LMU in Munich in 2000 (with Prof. J. Feldmann), a DAAD-fellow and later a research scientist in the group of Prof. $\mathrm{H}$. Weller and Dr. A. Eychmüller at the University of Hamburg in 2000-2005, and an IKERBASQUE-Fellow at the Center of Materials Physics, San Sebastian, Spain in 2011.

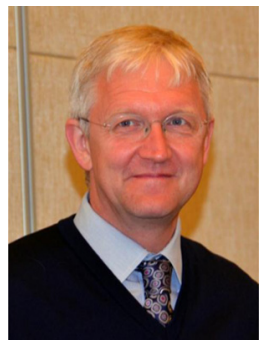

The academic career of Alexander Eychmüller started in Göttingen with studies of physics (Ph.D., University of Göttingen and MPI for Biophysical Chemistry, A. Weller and K.H. Grellmann) and continued at UCLA (postdoc with M.A. El-Sayed), Berlin (HMI with A Henglein), and the University of Hamburg (with $\mathrm{H}$. Weller). Since 2005 he holds a chair in Physical Chemistry at TU Dresden. His research interests include the synthesis and characterization of nanosized objects and their photophysical, electrochemical, and structural properties.

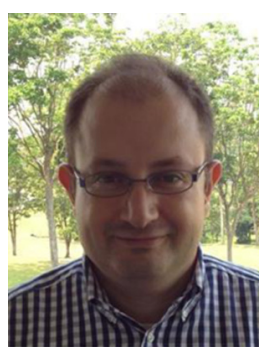

nocrystal optoelectronics.
Professor Hilmi Volkan Demir received his M.S. and Ph.D. degrees in electrical engineering from Stanford University, in 2000 and 2004, respectively. In 2004, he joined Bilkent University as a faculty member and is a Professor at the Department of Electrical-Electronics Engineering and the Department of Physics. He is concurrently Nanyang Professor at the School of Electrical Electronic Engineering and, Physical and Mathematical Sciences, NTU, and the Director of the LUMINOUS! Center of Excellence for Semiconductor Lighting and Displays. His current research interests include energy-saving LEDs for quality lighting, the science of excitonics for high-efficiency light harvesting, and na- 